\title{
ESTABLECIMIENTO Y VIGILANCIA DE ESTÁNDARES DE CALIDAD A TRAVÉS DE ISO
}

\author{
Juan Casiano González Guzmán*
}

\section{González-Guzmán J.C. El establecimiento y vigilancia de estándares de calidad a través de ISO. Hitos de Ciencias Económico Administrativas 2014;20 (57): 75-80. \\ RESUMEN}

El presente ensayo sirve como una guía para el proceso de estandarización y certificación de la calidad, en este caso, de la calidad microbiológica del agua purificada de la empresa Electropax.

Se exponen con detenimiento los pasos a seguir, empezando por la identificación de procesos de producción que pueden variar la calidad del producto final. Se revisan los requerimientos mínimos, las instituciones a consultar, las distintas vías de evaluación hasta llegar al proceso de certificación de calidad.

\section{González-Guzmán J.C. Establish and monitor quality standards through ISO-9000 Hitos de Ciencias Económico Administrativas 2014;20 (57): 75-80. ABSTRACT}

This paper serves as a guide to the process of standardization and quality certification, in this case, of the microbiological quality of purified water from the company Electropax.

The steps to follow are described in detail, starting with the identification of the production processes that can vary the quality of the final product. The minimum requirements are reviewed, the institutions that will be consulted, and the different ways to evaluate the steps until the quality certification process is reached.
Caso de Agua Purificada Electropax: Medición e implementación de estándares de calidad en la calidad microbiológica.

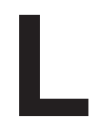

a competitividad de una empresa se emite principalmente por la calidad, ya sea del producto o servicio que ofrezca. Pero hablar de calidad no es un evento fortuito, no es cuestión de suerte o de orden aleatorio determinar que un producto o una empresa en sí tengan «mucha calidad». Asimismo se hace referencia a una estructura organizacional minuciosa, que hace atento caso de un protocolo de trabajo efectivo y confiable. Este protocolo de producción es quien da lugar a la implementación de estándares de calidad. No es que medir la calidad del producto final no sea suficiente prueba, sino que para lograr tal resultado hace falta revisar cada proceso y adecuarlo a su propio estándar.

Durante el proceso de purificación y envasado del agua ha sido medido desde hace ya varios años, de ahí que

\footnotetext{
"Maestro en Administración.
}

Fecha de recibido: 21 de febrero de 2014. Fecha de aceptación: 28 de marzo de 2014 . 
exista un sinnúmero de medidas internacionales que auxilien y certifiquen la calidad del agua purificada, uno de ellos es el bien conocido ISO-9001.

Implementar un Sistema de Gestión de la Calidad, conforme a los requisitos que establece la norma de calidad ISO 9001:2008, ayuda a resolver problemas prácticos. Esta certificación servirá para mejorar y estandarizar la calidad microbiológica del agua purificada, en este caso, producida por la empresa Electropax.

Los métodos de control de calidad de los alimentos, generalmente se basan en la inspección, pero esto no es suficiente para garantizar la seguridad de los mismos. En la Secretaría de Salud, para el control sanitario de los alimentos, se realizan visitas de verificación de las instalaciones, equipos y se observan las prácticas de higiene del personal para vigilar aquellos factores que funcionan como vehículos de peligros microbiológicos o fisicoquímicos en su elaboración. En estas verificaciones se toman muestras de ingredientes, del producto en proceso y del producto terminado para analizarlas microbiológica y fisicoquímicamente y los resultados obtenidos se comparan con las especificaciones que han sido establecidas en la legislación sanitaria.

Es importante empezar por ubicar los procesos que potencialmente pueden variar la calidad del agua. En la figura 1, se presenta un diagrama que muestra puntos clave durante el proceso de producción para mantener un nivel de calidad sin variaciones o con variaciones mínimas e imperceptibles.

Para medir los estándares de la calidad microbiológica del agua purificada Electropax, se utilizará El análisis de Riesgos, Identificación y Control de Puntos Críticos $(A R I C P C)$, el cual es un método efectivo de control de calidad microbiológica; dicho análisis se basa en dos puntos principales:

1) La identificación de aquellas operaciones en el proceso del alimento, en los cuales exista la posibilidad de que surjan desviaciones que puedan afectar negativamente la seguridad en la producción de alimentos, y;

Figura 1. Diagrama de causa y efecto

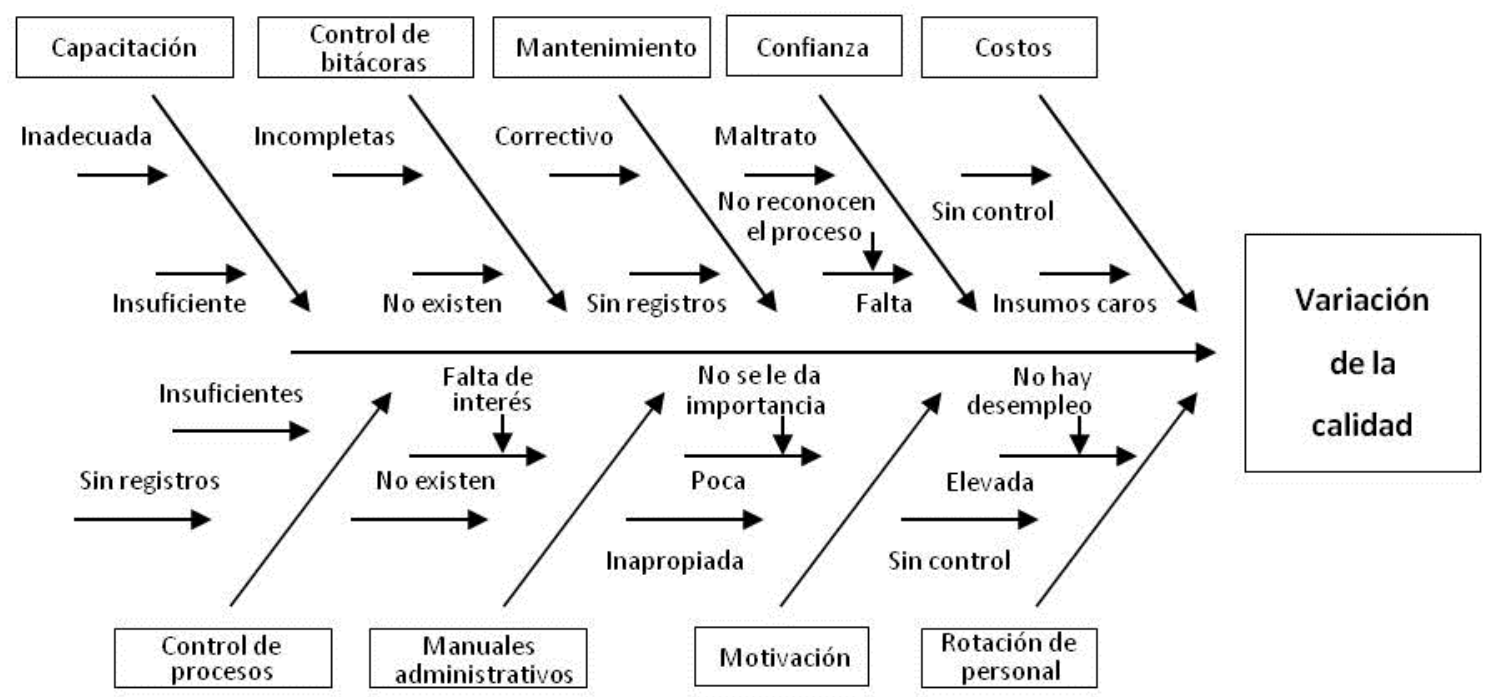


2) El desarrollo de acciones específicas que prevengan las posibles desviaciones antes que sucedan.

El ARICPC, es un método sistemático, racional y continuo de previsión y organización, con miras a lograr la seguridad de los alimentos, mejorar su calidad y disminuir las pérdidas ocasionadas por su alteración.

Este método puede ser aplicable a todas las operaciones del proceso de un alimento, desde la producción de la materia prima, la elaboración, su distribución y finalmente, la manipulación por el usuario final.

El ARICPC surge en la década de los sesenta, como un método para controlar los alimentos que se usarían en los programas espaciales, la aplicación de este método debía garantizar la seguridad de los alimentos que consumirían los astronautas.

De la misma manera, proporcionará una metodología que se enfoca hacia el modo en cómo deben evitarse o reducirse los peligros asociados a la producción de agua para consumo humano, por lo que es necesario realizar una evaluación cuidadosa de todos los factores internos y externos que intervienen en el proceso de purificación, desde la materia prima hasta el producto terminado, incluyendo la distribución y el consumo.

La medición de la calidad microbiológica del agua purificada y agua cruda, se hace en laboratorios públicos o privados o también puede hacerse en las instalaciones con reactivos que se compran en empresas con ese giro, (Secretaría de Salud, 1996).

\section{Requisitos Generales}

La organización debe establecer, documentar, implementar y mantener un sistema de gestión de la calidad y mejorar continuamente su eficacia de acuerdo con los requisitos de la Norma Oficial Mexicana NOM201-SSA1-2002.

\section{La organización debe:}

a) Determinar los procesos necesarios para el sistema de gestión de la calidad y su aplicación a través de la organización.

b) Determinar la secuencia e interacción de estos procesos.

c) Determinar los criterios y métodos necesarios para asegurarse que tanto la operación como el control de estos procesos sean eficaces.

d) Asegurarse de la disponibilidad de los recursos e información necesarios para apoyar la operación y el seguimiento de estos procesos.

e) Realizar el seguimiento, la medición cuando sea aplicable y el análisis de estos procesos, e;

f) Implementar las acciones necesarias para alcanzar los resultados planificados y la mejora continua de estos procesos.

\section{REQUISITOS DE LA DOCUMENTACIÓN}

\section{- Generalidades}

La documentación del Sistema de Gestión de la Calidad debe incluir:

a) Declaraciones documentadas de una política de la calidad y de objetivos de la calidad.

b) Un manual de la calidad.

c) Los procedimientos documentados y los registros requeridos por esta norma mexicana.

d) Los documentos, incluidos los registros que la organización determina que son necesarios para asegurarse de la eficaz planificación, operación y control de sus procesos.

\section{- Control de los documentos}

Los documentos requeridos por el sistema de gestión de la calidad deben controlarse. Los registros son un tipo especial de documento y deben controlarse de 
acuerdo con los requisitos mencionados en el control de los registros.

Debe establecerse un procedimiento documentado que defina los controles necesarios para:

a) Aprobar los documentos en cuanto a su adecuación antes de su emisión.

b) Revisar y actualizar los documentos cuando sea necesario y aprobarlos nuevamente.

c) Asegurarse que se identifican los cambios y el estado de versión vigente de los documentos.

d) Asegurarse que los documentos permanecen legibles y fácilmente identificables.

e) Asegurarse que los documentos de origen externo, que la organización determina que son necesarios para la planificación y la operación del sistema de gestión de la calidad, se identifican y que se controla su distribución. Y;

f) Prevenir el uso no intencionado de documentos obsoletos, y aplicarles una identificación adecuada en el caso que se mantengan por cualquier razón.

\section{- Control de los registros}

Los registros establecidos para proporcionar evidencia de la conformidad con los requisitos así, como de la operación eficaz del Sistema de Gestión de la Calidad deben controlarse. La organización debe establecer un procedimiento documentado para definir los controles necesarios para la identificación, el almacenamiento, la protección, la recuperación, el tiempo de retención y la disposición de los registros, (IMNC, ISO 9001:2008/ NMX-CC-9001-IMNC-2008, 2008).

\section{SOLICITUD DE CERTIFICACIÓN}

El departamento de certificación de sistemas, proporciona la guía de certificación de Sistemas de Gestión de la Calidad. Para iniciar formalmente el proceso de certificación, se deberá entregar al Instituto
Mexicano de Normalización y Certificación Asociación Civil, el FPEC03101- Solicitud y cuestionario de certificación de sistemas de gestión de la calidad ISO 9001:2008, vía fax, correo electrónico o personalmente en sus oficinas.

\section{- Análisis de la solicitud y cotización}

El Instituto Mexicano de Normalización y Certificación A.C., realiza el análisis de la solicitud del servicio considerando las exclusiones permisibles y justificadas para ISO 9001:2008/COPANT/ISO 9001:2008/ NMX-CC9001-INMC-2008 declaradas por la organización, el alcance del sistema de gestión de la calidad a certificar, las fechas solicitadas por la auditoría y el sitio o los sitios que abarcan la certificación. En esta etapa, el INMC podría solicitar información adicional.

Si la solicitud es viable, el INMC enviará la cotización del servicio de certificación de sistema de gestión de la calidad ISO 9001:2008/COPANT/ISO 9001:2008/ NMX-CC-9001-IMNC-2008y el Reglamento para marcas de IMNC-Certificación de sistemas de gestión.

Para el caso en que la solicitud no sea viable, el INMC envía una carta en donde explica las causas que impiden la viabilidad del proceso de certificación.

\section{- Firma del contrato}

Una vez aprobada la cotización del servicio de certificación de su sistema de gestión de la calidad, se firma el contrato de prestación de servicios de certificación y vigilancia de sistema de gestión de la calidad ISO 9001:2008/COPANT/ISO 9001:2008/NMXCC-9001-IMNC-2008.

\section{- Revisión documental en sitio}

Una vez aceptada la cotización y firmado el contrato, se deberá enviar al IMNC la documentación de su sistema con la documentación de soporte requerida, incluyendo:

a) El alcance del sistema de gestión de la calidad, incluyendo los detalles y la justificación de cualquier exclusión. 
b) Los procedimientos documentados establecidos para el sistema de gestión de la calidad o referencia a los mismos.

c) Una descripción de la interacción entre los procesos del sistema de gestión de la calidad.

d) Los resultados e informes de las auditorías internas, $y$;

e) Los resultados de la revisión por la dirección.
La revisión documental se hará en sitio con el objeto de:

a) Planificar y asignar recursos para hacer una revisión documental más profunda donde se requiera.

b)Proporcionar una oportunidad para retroalimentación inmediata o información para la organización.

c) Reunir la información necesaria referente al proceso, lugares o sitios del proveedor.

d) Acordar con este los detalles de la auditoría.

\section{REFERENCIAS}

Barra, R. (1985). Círculos de calidad en operación. México: McGraw Hill.

Besterfield Dale, H. (1995). Control de calidad. México: Prentice Hall.

Cantú Delgado, H. (2006). Desarrollo de una cultura de calidad. México: Mc Graw Hill.

Chiavenato, I. (1994). Iniciación a la organización y el control. México: McGraw Hill.

Chuen Tao, L. Y. (1980). El control de calidad en la empresa. España: Ediciones Deusto S.A.

Donald. (1981). Calidad. Mexico: CECSA.

Estévez Ramírez, F. (1999). Sistemas globales de gestión de calidad y ambiental. Las normas ISO 9000 e ISO 14000 del nuevo milenio. México: Qualitec Internacional, S.A. de C.V.

Evans, J. (s.f.). Administración y control de la calidad. México: International Thomson Editores.

Feigenbaum, A. V. (1994). Control Total de la Calidad. México: CECSA.

Folgar, O. (1996). Aseguramiento de la calidad ISO 9000. Argentina: Ediciones Macchi.

Gabor, A. (1990). Deming el hombre que descubrió la calidad. Argentina: Ediciones Granica S.A.

García, E. (1997). Metodología de la investigación. México: Nueva imagen.

Gibson, U. (1984). Manual de los pozos pequeños. Localización, diseño, construcción, uso y conservación. México: Limusa.

Goetsch, D. (1994). Introduction to total quality. USA: Inc. Merrill.
González Guzmán, J. (1996). Tesina: Proyecto de inversión para la producción y comercialización de chile habanero en Palenque Chiapas. México, Uniiversidad Veracruzana.

Hampton, D. (1989). Administración. México: McGraw Hill.

Harrington, H. (1993). Mejoramiento de los procesos de la empresa. Argentina: McGraw Hill.

IMNC. (2007). Guía de certificación de sistemas de gestión de la calidad. ISO 9001:2008 COPANT/ ISO 9001-2008 NMX-CC-9001-IMNC-2008. México. IMNC. (2008). ISO 9001:2005/NMX-CC-9000-IMNC2008. Sistemas de gestión de la calidad: Fundamentos y vocabulario, Viii. Instituto Mexicano de Normalización y Certificación A.C.

IMNC. (2008). ISO 9001:2008/NMX-CC-9001-IMNC2008. Sistemas de gestión de la calidad: Requisitos. México: Instituto Mexicano de Normalización y Certificación A.C.

Ishikawa, K. (1991). ¿Qué es el control de calidad? México : Prentice Hall.

Juran, J. (s.f.). Análisis y planeación de la calidad. México: McGraw Hill.

Karatsu, H. (1992). CTC: La sabidurpia japonesa. España: Gestión 2000 S.A.

Kasuga, H. (1997). Círculos de calidad. México: Grand. 


\section{UNIVHISTIDADJUARTI AUTÓNOMA DE TABASCO \\ "RSTUDIO EN LA DUDA. ACCIÓN EN LA FE"}

\section{DIVISIÓN ACADÉMICA CIENCIAS ECONÓMICO ADMINISTRATIVAS}

\section{DIPLOMADO}

LINEAMIENTOS Y FUNDAMENTOS METODOLÓGICOS PARA LA ELABORACIÓN DE TESIS EN LOS ESTUDIOS DE

\section{POSGRADOS}

Módulos:

1. Delimitación del Tema de Investigación.

2. Desarrollo del Marco de Referencia: Teórico y Contextual

3. Metodología de la Investigación.

4. Análisis y presentación de los resultados

Duración del diplomado: 160 horas

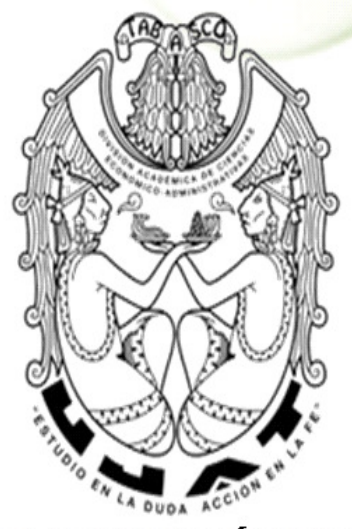

ÁREA de educación contunua
Requisitos:

1.Haber concluido satisfactoriamente el Plan de Estudios de los estudios de Posgrados de la DACEA.

2. Presentar protocolo de investigación.

3. Constancia de terminación de estudios.

4. Constancia de comprensión de textos en ingles emitida por el CEI

\section{¡INSCRIPCIONES ABIERTAS!}

\section{INFORMES:}

EDIFICIODEEDUCACIÓNCONTINUA Y A DISTANCIA

M.A. WILLIAM BALDEMARLÓPEZRODRIGUEZ

TELEFONO:358 1567 EXT.6219

Correo electrónico: educacioncontinua.dacea@ujat.mx 